\title{
A Geometric Proof of the Perron-Frobenius Theorem
}

\author{
ALBERTO BOROBIA † and UJUÉ R. TRías
}

ABSTRACT. We obtain an elementary geometrical proof of the classical PerronFrobenius theorem for non-negative matrices $A$ by using the Brouwer fixed-point theorem and by studying the dynamics of the action of $A$ on convenient subsets of $\mathrm{H}^{\text {r }}$.

\section{INTRODUCTION}

In this note $\mathbf{A}$ stands for a matrix, an endomorphism of $\boldsymbol{A}^{n}$, which has non-negative entries. Moreover, A will be irreducible (indecomposable), i.e., A cannot be put in the form

$$
\left({ }^{*}\right)\left(\begin{array}{c|c}
\mathbf{M} & 0 \\
\hline \mathbf{P} & \mathbf{N}
\end{array}\right) \quad \text { with } \mathbf{M} \text { and } \mathbf{N} \text { square matrices }
$$

by a re-ordering of the members of the canonical basis in $\boldsymbol{R}^{n}$.

A ray in $\mathfrak{A}^{n}$ (in the direction of $v \in \mathfrak{R}^{n} \backslash\{0\}$ ) will be the set $r[v]=\{\mu \nu / \mu>0\}$. We identify the collection of rays in $\mathfrak{R}^{n}$ with $S^{n-1}$.

Theorem I (Perron-Frobenius).-Let $\mathrm{A}$ be $a n \times n$ non-negative and irreducible matrix. Then there exists a simple positive eigenvalue $\lambda$ of $\mathbf{A}$ which has an associated positive eigenvector (i.e., all of whose coordinates are

† Partially supported by DGICYT PB89-0201

1991 Mathematics Subject Classification: $54 \mathrm{H} 20,15 \mathrm{~A} 48$.

Editorial Complutense. Madrid, 1992. 
positive), and which has the highest value among the moduli of the other eigenvalues of $\mathbf{A}$.

Written in this form, the theorem has some applications in geometry (for instance Vinberg in [V], section 2, uses it to obtain a description of acuteangled polytopes in Euclidean space and on the sphere). Based on this fact we decided to elaborate the following elementary geometrical proof (section 2) which should be well-known, though we were unable to find it in the literature. In section 3 we will complete the original version of the theorem with a hint of the proof of those parts not included above. Section 4 contains a historical note. We are indebted to professor J. M. Montesinos for proposing that we try to obtain such a proof, to professor Pierre de la Harpe for his helpful comments and to Pablo del Val for his assistance.

\section{PROOF OF THEOREM I}

(i) A acts on the set $\mathbf{R}^{+}$of non-negative rays, i.e., rays which emanate from the origin and lie in the quadrant

$$
\mathbf{C}^{+}=\left\{\left(x_{1}, . ., x_{n}\right) \in \mathfrak{R}^{n} / x_{i} \geq 0, i=1, \ldots, n\right\} .
$$

In 'fact, no ray in $\mathbf{R}^{+}$can be sent to zero. Otherwise, since $\dot{A}$ ' is nonnegative, it must have a column of zeros, which is not possible since $\mathbf{A}$ is irreducible.

(ii) No ray in $\partial \mathbf{R}^{+}$is left invariant by $\mathbf{A}$. Indeed, suppose there is a ray $r=r[v]$ lying in $\partial \mathbf{C}^{+}$for which $\mathbf{A}(r)=r$. Then, there exists $k, 0<k<n$, such that precisely the first $k$ coordinates of $v$ are zero (after a re-ordering of the basis if necessary). The condition $\mathbf{A}(r)=r$ now implies that $\mathbf{A}$ has the form $(*)$ where $\mathbf{M}$ is $k \times k$, and so $\mathbf{A}$ is not irreducible.

$$
1 \cdots \cdot
$$

(iii) Since $\mathbf{A}\left(\mathbf{R}^{+}\right) \subseteq \mathbf{R}^{+}$, the Brouwer's fixed point theorem asserts that there is an invariant ray $r$ in $\mathbf{R}^{+}$which, by (ii), must be positive.

Suppose $\pi$ is an invariant plane containing $r$. A acts in the circle. $S$ ! of rays lying in $\pi . \mathbf{R}_{i}^{+} \cap S$ ! is an arc $\mathbf{L}$ containing $r$ and by (i) $\mathbf{A}(\mathbf{L}) \subseteq \mathbf{L}$ :

(1) By (ii), $S^{1}$ is not pointwise fixed under $\mathbf{A}$.

(2) The set of points fixed by the action of $\mathrm{A}^{2}$ in $S^{1}$ does not consist only of $r$ and $-r$. Otherwise, the dynamics of the action of $A^{2}$ over $S^{1}$ would be as follows 


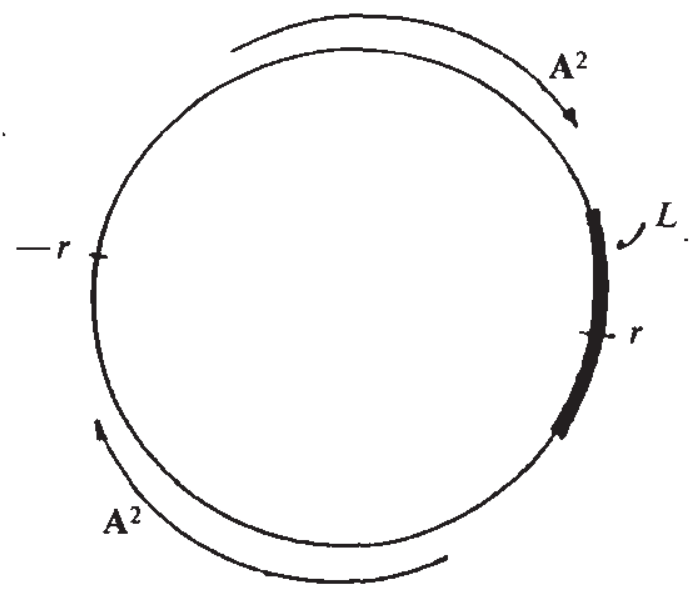

and then $A^{2}(L)$ would not be included in $L$, which is not possible.

From (1) we deduce the geometrical simplicity of the eigenvalue $\lambda$ corresponding to $r$, and from (2) the algebraic one.

Therefore, the eigenvalue $\lambda$ corresponding to $r$ is simple, positive and has an associated positive eigenvector $v_{\lambda}$.

(iv) It only remains to show that $\lambda \geq|\mu|$ for any other eigenvalue $\mu$. We argue by 'redutio ad absurdum' and, assuming that there is a $\mu$ such that $\lambda<|\mu|$, we distinguish two cases:

Case $1 .-\mu$ is real

Let $v_{\mu}$ be an eigenvector of eigenvalue $\mu$ such that its ray $r_{\mu} \notin \mathbf{R}^{+}$. Then $\mathbf{A}^{2}$ acts in the circle $S^{1}$ of rays lying in the plane generated by $\nu_{\curlywedge}$ and $\nu_{\mu}$, and fixes the set $\left\{ \pm r_{\lambda}, \pm r_{\mu}\right\}$. Since $\lambda<|\mu|$, the dynamics of the action of $\mathbf{A}^{2}$ on $S^{1}$ has two attracting points, $\left\{ \pm r_{\mu}\right\}$, and two repulsing points, $\left\{ \pm r_{\lambda}\right\}$. Then $r_{\mu}$ attracts one of the two points of $\partial \mathbf{R}^{+} \cap S^{1}$ out of $\mathbf{R}^{+}$, which is not possible.

Case $2 .-\mu$ is complex.

Let $\mathbf{P}$ be an invariant plane corresponding to the eigenvalue $\mu$. Then $\mathbf{P}$ does not contain any ray of $\mathbf{R}^{+}$. For otherwise the collection of rays of $\mathbf{R}^{+}$in $\mathbf{P}$ would be an arc $\mathbf{L}$ in the circle $S^{1}$ consisting of rays lying in $\mathbf{P}$. Since $\mathbf{A}$ is a rotation in $S^{1}, \mathbf{A}(\mathbf{L})$ would not be contained in $\mathbf{L}$ which contradicts that $\mathbf{A}\left(\mathbf{R}^{+}\right) \subseteq \mathbf{R}^{+}$. Now, consider the 3-space $\mathbf{E}^{3}$ generated by $\mathbf{P}$ and $\nu_{\lambda}$. As $|\mu|>\lambda$, the dynamics of the action of $\mathbf{A}$ in $\mathbf{E}^{3}$ shows a repulsing line generated by $v_{\lambda}$, and an attracting plane $\mathbf{P}$. This implies that the rays of $\mathbf{R}^{+} \backslash r_{\lambda}$ in $\mathbf{E}^{3}$ approach 
$\mathbf{P}$ as much as we want by repeated application of $\mathbf{A}$, and therefore we conclude that every such ray leaves $\mathbf{R}^{+}$after a number of applications of $\mathbf{A}$, which again is not possible because $\mathbf{A}\left(\mathbf{R}^{+}\right) \subseteq \mathbf{R}^{+}$(a formal proof of this is the following: let $r[x]$ be a ray of $\mathbf{E}^{3}$ in $\mathbf{R}^{+} \backslash r_{\lambda}$ with $x=v_{\lambda}+w$, where $w \in \mathbf{P}$ and $w \neq 0$. Then

$$
\mathbf{A}^{n}\left(\frac{x}{|\mu|^{n}}\right)=\frac{\mathbf{A}^{n}\left(v_{\Lambda}\right)+\mathbf{A}^{n}(w)}{|\mu|^{n}}=\frac{\lambda^{n}}{|\mu|^{n}} v_{\lambda}+\frac{\mu^{n}}{|\mu|^{n}} w .
$$

When $n \rightarrow \infty$ then $\frac{\lambda^{n}}{|\mu|^{n}} \rightarrow 0$, and hence the ray $\mathbf{A}^{n}(r[x])$ can come as close to $\mathbf{P}$ as we want, because the modulus of $\frac{\mu^{n}}{|\mu|^{n}} w \in \mathbf{P}$ is constant)

\section{A SKETCH OF THE PROOF OF THE ORIGINAL STATEMENT OF PERRON-FROBENIUS THEOREM}

Frobenius in [F2] proved a generalization of Perron's theorem for irreducible matrices. One part of his theorem is our theorem I. In what follows we state the rest of the original version (theorem II below) and give a sketch (also of elementary nature) of its proof.

A non-negative matrix $\mathbf{B}$ is primitive if there exists a natural number $j \geq 1$ such that $\mathbf{B}^{j}$ is positive (i.e., all entries of $\mathbf{B}^{j}$ are positive).

Let $\left\{e_{1}, e_{2}, \ldots, e_{n}\right\}$ be the canonical basis of $\mathcal{A}^{n}$, and let $r_{i}$ be the ray $r\left[e_{i}\right]$ for $i=1,2, . ., n .\left\{r_{1}, r_{2}, \ldots, r_{n}\right\}$ are the vertices of the simplex $\mathbf{R}^{+}$. In the next definition the term "coordinates" means barycentric coordinates with respect to the vertices of the simplex $\mathbf{R}^{+}$: if $r \in \mathbf{R}^{+}$has exactly $t$ positive coordinates we will say that $r$ has dimension $t, \operatorname{dim}[r]=t$.

Theorem II (Frobenius 1912), - Let A be a $n \times n$ non-negative and irreducible matrix, then by a re-ordering of the members of the canonical basis of $\mathbf{R}^{n}$, $\mathbf{A}$ can be put in the form

$$
\overline{\mathbf{A}}=\left|\begin{array}{ccccc}
0 & 0 & \ldots & 0 & \mathrm{~A}_{d} \\
\mathrm{~A}_{1} & 0 & \ldots & 0 & 0 \\
0 & \mathrm{~A}_{2} & \ldots & 0 & 0 \\
. & . & \ldots & . & . \\
0 & 0 & \ldots & \mathrm{A}_{d-1} & 0
\end{array}\right|
$$


where the blocks on the diagonal are square zero matrices of possibly different sizes and $\overline{\mathrm{A}}^{d}$ is a direct sum of d primitive matrices.

A sketch of the proof: We suppose that rang $\mathbf{A}=n$ (otherwise the argument is slightly more elaborated), then the lemmas below give the proof:

Lemma 1: Let $r$ be a ray in $\mathbf{R}^{+}$, then $\operatorname{dim}[\mathbf{A}(r)] \geq \operatorname{dim}[r]$. Hence there exists an integer $s \geq 1$ such that $\forall s^{\prime} \geq s$ and $\forall r \in \mathbf{R}^{+} \operatorname{dim}\left[\mathbf{A}^{s^{\prime}}(r)\right]=\operatorname{dim}\left[\mathbf{A}^{s}(r)\right]$.

Proof of lemma 1: Since rang $\mathbf{A}=n$; if $\operatorname{dim}[\mathbf{A}(r)]<\operatorname{dim}[r]$ then a neighbourhood of $r$ in $\mathbf{R}^{+}$leaves $\mathbf{R}^{+}$under the action of $\mathbf{A}$, which is not possible. For instance, suppose $\mathbf{R}^{+}$is a triangle, $\operatorname{dim}[r]=2$ and $\operatorname{dim}[\mathbf{A}(r)]=1$. Then each neighbourhood of $r$ in $\mathbf{R}^{+}$contains a half disk of small radius that, under the action of $\mathbf{A}$, necessarily leaves $\mathbf{R}^{+}$. Moreover, for any integer $j \geq 1$ the function that associates the integer $\operatorname{dim}\left[\mathbf{A}^{j}(r)\right]$ to the ray $r \in \mathbf{R}^{+}$is constant in the interior of each face of $\mathbf{R}^{+}$. Since $\operatorname{dim}[r] \leq n \forall r \in \mathbf{R}^{+}$we deduce the last sentence in lemma 1

Lemma 2: There exists an integer $t \geq 1$ such that $\operatorname{dim}\left[\mathbf{A}^{s}\left(r_{i}\right)\right]=t$ for $i=1, . ., n$.

Proof of lemma 2: Otherwise we would have a partition of the set $\left\{r_{\mathrm{l}}, . ., r_{n}\right\}$ depending on the dimension of $\mathbf{A}^{s}\left(r_{i}\right)$, and $\mathbf{A}$ would be irreducible

Lemma 3: The (t-1)-dimensional faces of $\mathbf{R}^{+}$contain in their interior either zero or $t$ elements of $\left\{\mathbf{A}^{s}\left(r_{1}\right), \mathbf{A}^{s}\left(r_{2}\right), \ldots, \mathbf{A}^{s}\left(r_{n}\right)\right\}$. The faces that contain $t$ elements are disjoint.

Proof of lemma 3: Assume there are two ( $t-1)$-dimensional faces of $\mathbf{R}^{+}$ such that the number of elements of $\left\{\mathbf{A}^{s}\left(r_{1}\right), \ldots, \mathbf{A}^{s}\left(r_{n}\right)\right\}$ in each are both greater than zero but different. Then arguing like in lemma $2 \mathrm{~A}$ would be irreducible. Thus each ( $t$-1)-dimensional face in $\mathbf{R}^{+}$contains either zero or $v$ elements. If $v>t$ then rang $\mathrm{A}<n$; but $v<t$ contradicts lemma 1 . Then $v=t$ and we again use rang $\mathbf{A}=n$ to finish

Let $D$ be the set of these $d=n / t$ faces. From lemma 1 and lemma 3 we deduce that as $\mathbf{A}$ is irreducible then $\mathbf{A}$ acts in $\boldsymbol{R}^{n}$ producing a cyclic permutation of the $d$ vectorial subspaces of dimension $t$ that contain some element of $D$. That is, re-ordering the members of $\left\{e_{1}, e_{2}, \ldots, e_{n}\right\}$ the matrix $\mathbf{A}$ 
is replaced by $\overline{\mathbf{A}}$. $\overline{\mathbf{A}}^{d}$ will be the direct sum of $d t \times t$ non-negative matrices, and they are primitives by lemma 2

\section{HISTORICAL NOTE}

The first proof of this theorem, for positive matrices, is the analytic one given by Perron [P] in 1907. Shortly afterwards, Frobenius, in series of papers $[\mathrm{F} 1]$ and $[\mathrm{F} 2]$, extended the result for non-negative matrices and proved it in a purely algebraic way. Alexandroff and Hopf [A-H] in 1935 and Debreu and Herstein [D-H] in 1953 published new topological proofs of parts of the classical theorem, using the fixed-point theorem of Brouwer in a similar way that we use here. Samelson [Sa] in 1956 gave, for positive matrices, a proof of the existence of exactly one positive eigenvector defining a metric in the interior of $\mathbf{R}^{+}$and then showing that $\mathbf{A}$ contracts $\mathbf{R}^{+}$. Different constructions are those of Wieland [W] and Brauer [B], who again employ algebraic and analytic techniques. Nowadays, some of these proofs are reproduced with slight modifications in some books: see, for instance, [G] and $[\mathrm{Pu}]$.

\section{References}

[A-H] Alexandroff, P. und Hopf, H.: Topologie. J. Springer, Berlin, 1935. Reprinted by Chelsea, New York, 1965. See pages 480-481.

[B] BRAUER, A.: A new proof of theorems of Perron and Frobenitus on nonnegative matrices. Duke Math. J., 24 (1957), 367-378.

[D-H] Debreu, G. and Herstein, I. N.: Nonnegative square matrices. Econometrica, 21 (1953), 514-518.

[F1] FrobENIUS, G.: Über Matrizen aus positiven Elementen, I and II. Sitzungsberischte der koniglich preussischen Akademie der Wissenschaften zu Berlin (1908), 471-476 and (1909), 514-518.

[F2] Frobenius, G.: Über Matrizen aus nicht negativen Elementen. Sitzungsberichte der königlich Preussischen Akademie der Wissenschaften zu Berlin (1912), 456-477.

[G] GRAHAM, A.: Nonnegative matrices and applicable topics in linear algebra. Ellis Horwood, Chichester, 1987.

[P] PERRON, O.: Zur Theorie der Matrizen. Mathematische Annalen, 64 (1907), 248-263.

[Pu] Pullman, N. J.: Matrix Theory and its Applications. Marcel Dekker, New York and Basel 1976, 102-117.

[Sa] Samelson, H:: On the Perron-Frobenitus theorem. Michigan Math., 4 (1956), 57-59. 
[V] VINBERG, E. B.: Hyperbolic reflection groups. Russian Math. Surveys, 40 (1985), 31-75.

[W] WIELAND, H.: Unzerlegbare nicht negative Matrizen. Mathematische Zeitschrift, 52 (1950), 642-648.

Dpto. Matemáticas

U.N.E.D.

28040 Madrid, SPAIN

Dpto. Geometria y Topologia

Universidad Complutense

Recibido: 4 de febrero de 199I

28040 Madrid, SPAIN Revisado: 25 de mar7o de 199 I 